\title{
Identification of a Novel Fumarate Reductase Potentially Involved in Electron Bifurcation Jeroen Girwar Koendjbiharie ${ }^{1}$, Richard van Kranenburg ${ }^{1,2 *}$
}

1 Corbion, Gorinchem, Netherlands

2 Wageningen University, Laboratory of Microbiology, Wageningen, Netherlands

* Correspondence: richard.van.kranenburg@corbion.com

\begin{abstract}
In the succinic acid-producing bacterium Pseudoclostridium thermosuccinogenes the fumarate reductase (FRD) genes reside in an operon together with those encoding an electron bifurcating complex (FIXABCD$\mathrm{HdrABC}$ ) that shuttles electrons from NADH to ferredoxin and a disulfide bond. Based on phylogeny and genomic co-occurrence we propose two hypothetical mechanisms via which the FRD is involved in electron bifurcation: (I) A disulfide bond from a hitherto unknown cofactor is reduced by the electron-bifurcating FIXABCD-HdrABC complex, using NADH to generate two thiol groups, while facilitating the unfavourable reduction of ferredoxin by NADH. The disulfide bond is subsequently regenerated via the reduction of fumarate by the FRD using the previously formed thiol groups. (II) The FRD forms an integral part of the FIXABCD-HdrABC complex, and NADH is used to reduce ferredoxin and fumarate directly, without an intermediate disulfide-forming cofactor. Either way enables the conservation of additional energy by a soluble FRD, analogous to fumarate respiration.
\end{abstract}

Keywords: Pseudoclostridium thermosuccinogenes; fumarate reductase; FRD; electron-bifurcation 


\section{Hypothesis}

\section{Introduction}

Pseudoclostridium thermosuccinogenes is the only thermophile known to produce succinic acid as one of its main products of fermentation, together with acetic acid, formic acid and several minor products (1-3). Succinic acid is a versatile molecule that can form one of the core building blocks in a bio-based economy, where it is produced from biomass, as an alternative to petroleum (4). From an industrial point of view it can be advantageous to use a thermophilic production organism (5). Therefore, we set out to identify and characterize the enzymes responsible for succinic acid production in $P$. thermosuccinogenes, as this might facilitate future engineering of thermophilic succinic acid cell-factories.

RNA-seq. data of a previous study led to the identification of set of genes that likely form an operon (Figure 1 ), starting with the gene encoding a fumarate hydratase, the enzyme responsible for the interconversion of malate and fumarate (3). This operon further contains a gene homologous to the flavoprotein subunit of fumarate reductase/succinate dehydrogenase (FrdA), the core subunit that is shared between all known fumarate reductases and succinate dehydrogenases (6), and the only isoform of it present in $P$. thermosuccinogenes. Surprisingly, eight more genes are present in between the fumarate hydratase and the fumarate reductase flavoprotein subunit. The last of these, upstream of $f r d A$, encodes a protein that is homologous to ferredoxins and most likely represents the iron-sulfur subunit of the suspected fumarate reductase (FrdB). The other seven genes appear to encode a flavin oxidoreductase-heterodisulfide reductase complex (FIXABCD-HdrABC), a large enzyme complex involved in flavin-based electron bifurcation that appears to be widespread in anaerobic bacteria (7). Lastly, a small hypothetical membrane protein-encoding gene is present in the operon, directly downstream of frdA. The small membrane protein contains a so-called PQ-loop motif, which is found in a specific (newly described) family of membrane transporters, suggesting that it might be a succinic acid transporter (8).

Following phylogenetic evidence and the co-occurrence of genes responsible for succinate production with this electron bifurcating FIXABCD-HdrABC complex in one operon, we propose a hypothesis for the mechanisms by which succinic acid is formed in $P$. thermosuccinogenes, presented further below. First, fumarate reductases will be introduced in more detail, as well as flavin-based electron bifurcation, focussing in particular on the FIXABCD-HdrABC complex.

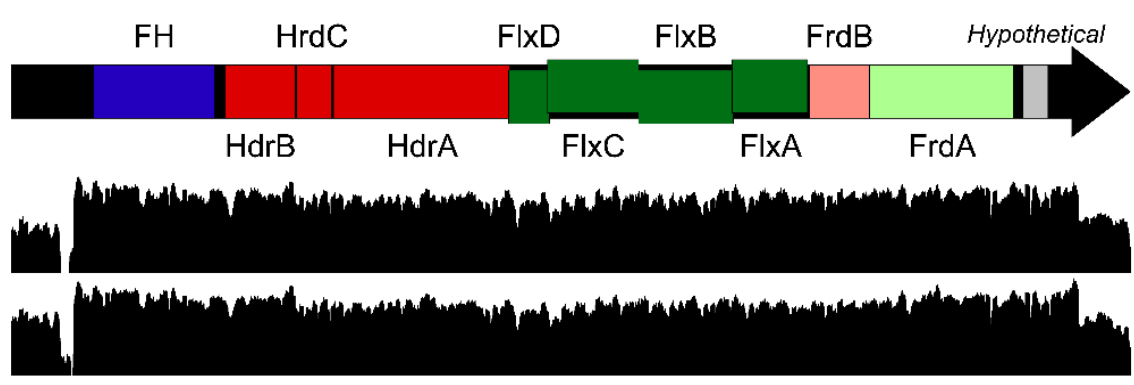

Figure 1. Schematic depiction of the 'succinic acid operon' in $P$. thermosuccinogenes, with below it the (log10) transcription coverage during growth on glucose (upper track) and on xylose (lower track), from data generated previously (3). Indentations reflect overlap with the preceding open reading frame. FH: fumarate hydratase, Hdr: heterodisulfide reductase, FIx: flavin oxidoreductase, Frd: fumarate reductase. Locus tags of genes are from left to right: CDQ83_03355, CDQ83_03350，CDQ83_03345，CDQ83_03340，CDQ83_03335，CDQ83_03330，CDQ83_03325, CDQ83_03320, CDQ83_03315, CDQ83_03310,CDQ83_03305.

\section{Fumarate reductases}

The various enzymes responsible for the interconversion of fumarate and succinate (i.e. fumarate reductases and succinate dehydrogenases, FRD/SDH) are diverse in their subunit configurations, but are all part of a larger protein superfamily based on a homologous flavoprotein, which also includes L-aspartate oxidases, anaerobic glycerol-3-phosphate dehydrogenases, and adenylyl-sulfate reductases (6). FRD/SDHs can be divided in two main groups: soluble and membrane-bound. The latter are part of the electron 
transport chain, and thus often linked to proton translocation, and are generally referred to as the succinate:quinone oxidoreductase superfamily (Figure 2) (9). Five types (i.e. A-E) of the membrane bound succinate:quinone oxidoreductase superfamily are distinguished, containing two anchor domains, FrdC and FrdD (which are fused in type $B$ ), together with the flavoprotein subunit (FrdA) and the iron-sulfur subunit (FrdB) (9). In Type $E$, which is only found in archaea, the anchor domains are unrelated to those of the other types, and are therefore referred to as FrdE and FrdF.

Only a handful of soluble FRDs have been described and they are diverse in their function and their electron donor, as well as in the additional subunits associated with FrdA. The soluble FRD in methanogenic archaea is thiol-driven, as it uses the cofactors COM-SH and CoB-SH to form succinate together with the CoM-S-S$\mathrm{CoB}$ heterodisulfide. It resembles type $\mathrm{E}$ of the succinate:quinone oxidoreductase superfamily, and the iron-sulfur subunit (FrdB) is essentially a fusion of FrdB with FrdE from the membrane-bound type E (10, 11). The soluble FRDs described in the eukaryotes Saccharomyces cerevisiae and Trypanosoma brucei use $\mathrm{NADH}$ as electron donor, and are monomeric proteins, consisting only of $\operatorname{FrdA}(12,13)$. The soluble FRD from Shewanella species is also monomeric, and is present in the periplasm, where it accepts electrons from a c-type cytochrome $(14,15)$. Finally, the soluble FRD from Aquificae, chemolitoautotrophs that use the reverse TCA-cycle to fix $\mathrm{CO}_{2}$, has 5 subunits and uses $\mathrm{NADH}(16)$. Its FrdA and FrdB are closely related to those of the membrane bound FRD/SDHs, but the other three subunits are unique. Figure 2 shows an overview of the configurations of the different known FRD/SDHs.

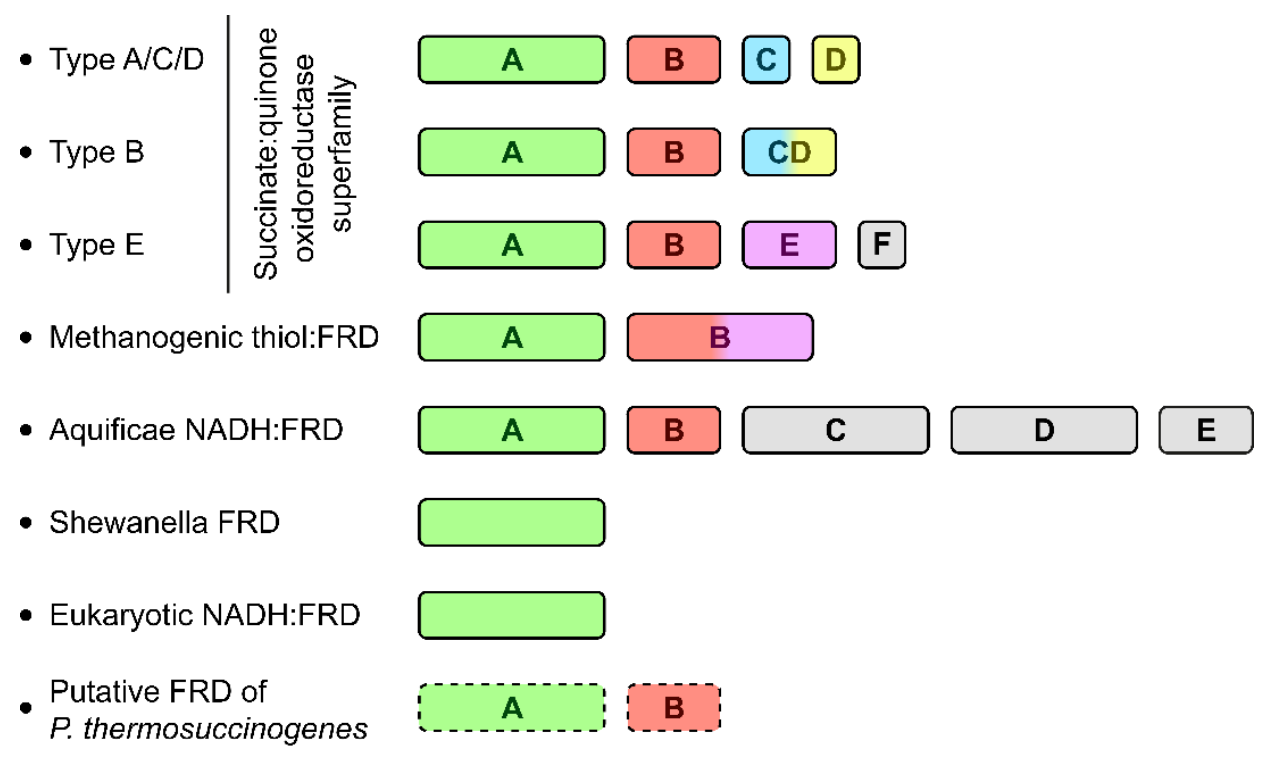

Figure 2. List of the known fumarate reductases, including the putative fumarate reductase of $P$. thermosuccinogenes. A schematic overview of the subunits that are part of each type is shown. The colours indicate homologous proteins or protein domains; the grey subunits are unique to that type of fumarate reductase. Note that the letter annotations used for the Aquificae fumarate reductase subunits C, D, and E do not to reflect homology with the corresponding annotations of the succinate:quinone oxidoreductase superfamily. Figure adapted from Jardim-Messeder et al. (2017) (6).

\section{Flavin-based electron bifurcation}

Electron bifurcation refers to the phenomenon where a hydride electron pair is split into one electron with a more negative reduction potential and another with a more positive reduction potential. As such, the reducing power of one is amplified at the expense of the other (17). Note that a more negative reduction potential means a higher potential energy.

Two variants of electron bifurcation are known to occur in nature: Quinone-based electron bifurcation (QBEB) and flavin-based electron bifurcation (FBEB). QBEB occurs in complex III of the electron transport chain, where the electron pair of the quinone is split between cytochrome $b$ (low-potential) and cytochrome c (high-potential). FBEB only occurs in strictly anaerobic prokaryotes. A flavin (i.e. FAD or FMN) is essential as a prosthetic group and the low-potential acceptor is always a ferredoxin or a flavodoxin, but a wide 
range of different high-potential acceptors and two-electron donors have been described (Figure 3). The low-potential ferredoxin or flavodoxin (generated via FBEB) can subsequently be used in processes where the reduction potential of the two-electron donor (e.g. NADH) is not sufficiently low, such as reduction of $\mathrm{N}_{2}$ to $\mathrm{NH}_{3}$ or $\mathrm{CO}_{2}$ to $\mathrm{CO}(17,18)$.

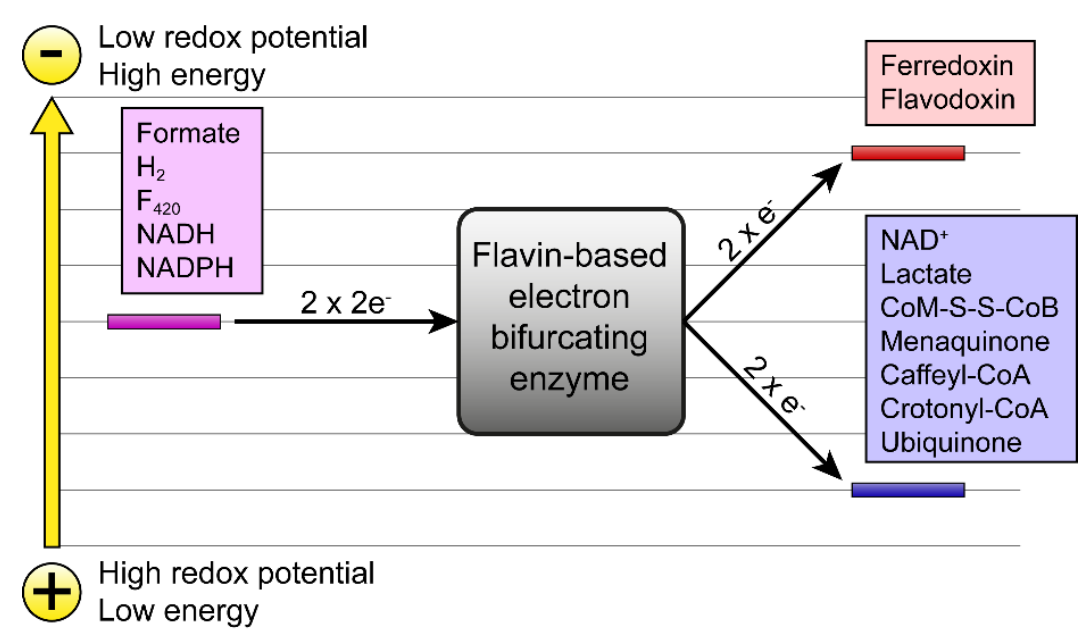

Figure 3. All the currently known electron donors and acceptors involved in flavin-based electron bifurcation (FBEB). Figure was adapted from Buckel and Thauer (2018) (17).

\section{The FlxABCD-HdrABC complex}

The predicted seven-subunit flavin oxidoreductase-heterodisulfide reductase (FIXABCD-HdrABC) complex is composed of two parts: A three-subunit heterodisulfide reductase part, and a four-subunit flavin oxidoreductase part. In vitro evidence regarding the function of the full complex is lacking, but in vivo results from Desulfovibrio vulgaris strongly support the proposed function as a bifurcation complex coupling the energetically unfavourable reduction of ferredoxin by NADH to the favourable reduction of a disulfide bond (Figure S1A) (7). This is analogous to a similar complex in hydrogenotrophic methanogenic archaea, which also contains the three-subunit heterodisulfide reductase part, but in that case together with a threesubunit [NiFe]-hydrogenase part, i.e. MvhADG-HdrABC (19). The complex in archaea uses hydrogen to reduce ferredoxin, also by coupling it to a simultaneous reduction of a disulfide bond; namely, that of the CoM-S-S-COB heterodisulfide (Figure S1B). MvhADG-HdrABC has been studied in vitro and recently a crystal structure was published (20). In Desulfovibrio vulgaris, a small protein, DsrC presumably acts as the disulfide electron acceptor, via internal disulfide bonds of cysteine residues $(7,21)$. The FIXABCDHdrABC complex is widespread in anaerobic bacteria, but DscR is only conserved in organisms with a dissimilatory sulfur metabolism, where it is often located next to the FIXABCD-HdrABC complex, and is used for reduction of sulfite. It is likely that other disulfide bond-containing compounds or proteins acts as the electron acceptor in other bacteria that possess the FIXABCD-HdrABC complex.

\section{Fumarate reductase in $P$. thermosuccinogenes}

FrdA of $P$. thermosuccinogenes is related to the soluble FRDs, following the results from a phylogenetic tree that was constructed using sequences of enzymatically studied FRDs, shown in Figure S2A. The soluble FRDs do not form a tight cluster, unlike the membrane bound FRDs of the succinate:quinone oxidoreductase superfamily. By directly comparing the FrdA sequence of $P$. thermosuccinogenes with those of the two closest branching (enzymatically-studied) homologs from Methanothermobacter thermoautotrophicus and Shewanella frigidimarina, it is clear that it is more similar to the former, i.e. the archaeal thiol-driven FRD. A complementary picture emerges from the phylogenetic tree of the corresponding iron-sulfur (FrdB) subunits (Figure S2B).

The succinic acid operon in P. thermosuccinogenes is not conserved within the other Hungateiclostridiaceae, except in Ruminiclostridium josui, which contains all the genes, save for the class II fumarate hydratase. An unrelated class I fumarate hydratase is present instead. The genes in the operon of $P$. 
thermosuccinogenes appear to be most homologous to those from the three known Christensenella species, suggesting that the operon has been acquired via lateral gene transfer.

The STRING database for protein-protein association networks was consulted to predict functional partners, using FrdA of $R$. josui as the query ( $P$. thermosuccinogenes was not included in the STRING database) (22). Scoring based on genomic neighborhood and co-occurrence, or both parameters alone led in all three instances to the selection of FrdB together with the seven subunits of the FIXABCD-HdrABC as predicted functional partners with (very) high confidence. The small hypothetical membrane protein is also selected, but with a significantly lower score, suggestive of a less stringent functional relationship. The latter supports the possibility that it represents a novel succinate transporter, as this functionality should be easily replaceable - thus explaining a less stringent co-occurrence. Alternatively, the membrane protein could also be a membrane anchor analogous to succinate:quinone oxidoreductase superfamily, but without any direct enzymatic role in the FRD reaction. However, based on the presence of the PQ-loop, we hypothesise that CDQ83_03300 is a specific succinic acid transporter.

Based on the strong genomic association of the putative FRD in $P$. thermosuccinogenes with the FIXABCDHdrABC complex, and the relative similarity of FrdA and FrdB with those of thiol (CoM/CoB) driven FRDs from methanogenic archaea, we propose the following two hypothetical mechanisms by which succinic acid is formed in $P$. thermosuccinogenes:

1. Two thiol groups, either from a specific cofactor (pair) or from a (small) protein are used by FRD to reduce fumarate, forming succinate. The disulfide bond formed is than reduced by the electronbifurcating FIXABCD-HdrABC complex, using NADH, in order to regenerate the original two thiol groups, while facilitating the otherwise unfavourable reduction of ferredoxin by NADH (Figure 4A).

2. The FRD is an integral part of the electron-bifurcating FIXABCD-HdrABC complex, and NADH is directly used to reduce fumarate and ferredoxin, without an additional disulfide forming cofactor/protein (Figure 4B).

A

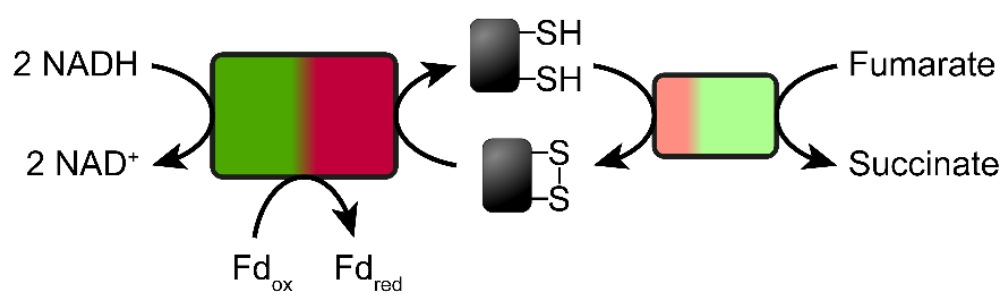

B

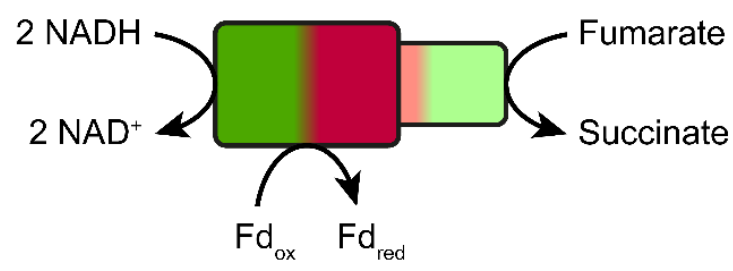

Figure 4. Two proposed hypothetical mechanisms for succinate production in $P$. thermosuccinogenes; (A) either involving an intermediate thiol/disulfide cofactor, which could be both a small molecule (pair) or a peptide, or (B) involving the direct electron transfer from NADH to fumarate, coupled to the simultaneous reduction of ferredoxin.

In principle, it should also be possible for NADH to function directly as the cofactor for FRD, without any electron bifurcation, as is the case with many of the other soluble FRDs. However, that would make the strong association with the FIXABCD-HdrABC complex a rather odd coincidence. Furthermore, the known $\mathrm{NADH}$-dependent FRDs are not involved in central energy metabolism, while $P$. thermosuccinogenes, a strictly anaerobic fermentative organism and likely to be constrained by its ability to generate metabolic energy, produces succinic acid as one of its main products of fermentation. It seems therefore credible that it can couple succinic acid production to additional energy conservation, in the form of reduced 
ferredoxin, as we hypothesize. Sridhar et al. (2000) (23) reported NADH-dependent reduction of fumarate in cell-free extract of $P$. thermosuccinogenes, but did not present their data. We were unable to reproduce this finding.

Something very similar was proposed recently for the reverse TCA-cycle of sulfur-oxidizing bacteria found in symbiosis with tubeworms (24). In those bacteria, the HdrABC-FIXABCD complex colocalizes on the genome with a FRD (homologous to the methanogenic thiol-driven FRD, TfrAB) as well as a 2oxoglutarate:ferredoxin oxidoreductase (KorABCD). The authors propose that the electrons from NADH are transferred to fumarate and succinyl-CoA (forming succinate and 2-oxoglutarate), either directly via the existence of a very large electron bifurcation complex (HdrABC-FIXABCD-KorABCD-TfrAB), or via the mediation of ferredoxin and/or DsrC, formed by HdrABC-FIxABCD.

If indeed the reduction of fumarate is connected to the reduction of ferredoxin, the net reaction will be: Fumarate $+2 N A D H+F d \rightarrow$ Succinate $+2 N A D^{+}+F d^{2-}$. When the reduced ferredoxin is used for proton export, via $\mathrm{NAD}^{+}$reduction in the Rnf complex (which is present in $P$. thermosuccinogenes), the net reaction becomes Fumarate $+N A D H \rightarrow$ Succinate $+N A D^{+}+\Delta \mu_{H^{+}}$, quite like anaerobic fumarate respiration (25), except: (I) Only two protons (or sodium ions) are pumped by the Rnf complex per reduced fumarate, compared to the four protons pumped by Type $1 \mathrm{NADH}$ dehydrogenase, and (II) ferredoxin (vs. quinone) allows for more flexibility, since it can be used in a multitude of processes that are not just membrane associated.

Future experimental evidence should show whether or not the putative FRD is involved in electron bifurcation, and whether this proceeds indirectly, via an intermediate thiol/disulfide cofactor (Figure 4A), or whether it perhaps is coupled directly to the reduction of ferredoxin by NADH (Figure 4B), and thus whether fumarate needs to be added to the ever-growing list of high-redox-potential acceptors involved in FBEB.

\section{Funding}

This research was funded by Corbion and the European Union Marie Skłodowska-Curie Innovative Training Networks (ITN), contract number 642068.

\section{Conflicts of Interest}

The authors declare no conflict of interest. 
1. Drent WJ, Lahpor G a, Wiegant WM, Gottschal JC. 1991. Fermentation of Inulin by Clostridium thermosuccinogenes sp. nov., a Thermophilic Anaerobic Bacterium Isolated from Various Habitats. Appl Environ Microbiol 57:455-462.

2. Sridhar J, Eiteman M a. 1999. Influence of Redox Potential on Product Distribution in Clostridium thermosuccinogenes. Appl Biochem Biotechnol 82:91-102.

3. Koendjbiharie JG, Wiersma K, van Kranenburg R. 2018. Investigating the Central Metabolism of Clostridium thermosuccinogenes. Appl Environ Microbiol 84:e00363-18.

4. Bozell JJ, Petersen GR. 2010. Technology development for the production of biobased products from biorefinery carbohydrates - the US Department of Energy's "Top 10" revisited. Green Chem 12:539-554.

5. Zeldes BM, Keller MW, Loder AJ, Straub CT, Adams MWW, Kelly RM. 2015. Extremely thermophilic microorganisms as metabolic engineering platforms for production of fuels and industrial chemicals. Front Microbiol 6:1209.

6. Jardim-Messeder D, Cabreira-Cagliari C, Rauber R, Turchetto-Zolet AC, Margis R, Margis-Pinheiro M. 2017. Fumarate reductase superfamily: A diverse group of enzymes whose evolution is correlated to the establishment of different metabolic pathways. Mitochondrion 34:56-66.

7. Ramos AR, Grein F, Oliveira GP, Venceslau SS, Keller KL, Wall JD, Pereira IAC. 2015. The FIxABCDHdrABC proteins correspond to a novel NADH dehydrogenase/heterodisulfide reductase widespread in anaerobic bacteria and involved in ethanol metabolism in D esulfovibrio vulgaris Hildenborough. Environ Microbiol 17:2288-2305.

8. Feng L, Frommer WB. 2016. Evolution of Transporters: The Relationship of SWEETs, PQ-loop, and PnuC Transporters. Trends Biochem Sci 41:118-119.

9. Lemos RS, Fernandes AS, Pereira MM, Gomes CM, Teixeira M. 2002. Quinol:fumarate oxidoreductases and succinate:quinone oxidoreductases: phylogenetic relationships, metal centres and membrane attachment. Biochim Biophys Acta - Bioenerg 1553:158-170.

10. Bobik TA, Wolfe RS. 1989. An unusual thiol-driven fumarate reductase in Methanobacterium with the production of the heterodisulfide of coenzyme $\mathrm{M}$ and $\mathrm{N}$-(7-mercaptoheptanoyl)threonine-O3-phosphate. J Biol Chem 264:18714-8.

11. Heim S, Kunkel A, Thauer RK, Hedderich R. 1998. Thiol : fumarate reductase (Tfr) from Methanobacterium thermoautotrophicum . Identification of the catalytic sites for fumarate reduction and thiol oxidation. Eur J Biochem 253:292-299.

12. Kim S, Kim CM, Son Y-J, Choi JY, Siegenthaler RK, Lee Y, Jang T-H, Song J, Kang H, Kaiser CA, Park HH. 2018. Molecular basis of maintaining an oxidizing environment under anaerobiosis by soluble fumarate reductase. Nat Commun 9:4867.

13. Coustou V, Besteiro S, Rivière L, Biran M, Biteau N, Franconi J-M, Boshart M, Baltz T, Bringaud F. 2005. A Mitochondrial NADH-dependent Fumarate Reductase Involved in the Production of Succinate Excreted by Procyclic Trypanosoma brucei. J Biol Chem 280:16559-16570.

14. Myers CR, Myers JM. 1992. Fumarate reductase is a soluble enzyme in anaerobically grown Shewanella putrefaciens MR-1. FEMS Microbiol Lett 98:13-19.

15. Schwalb C, Chapman SK, Reid GA. 2002. The membrane-bound tetrahaem c-type cytochrome CymA interacts directly with the soluble fumarate reductase in Shewanella. Biochem Soc Trans 30:658-62.

16. Miura A, Kameya M, Arai H, Ishii M, Igarashi Y. 2008. A soluble NADH-dependent fumarate reductase in the reductive tricarboxylic acid cycle of Hydrogenobacter thermophilus TK-6. J Bacteriol 190:7170-7.

17. Buckel W, Thauer RK. 2018. Flavin-Based Electron Bifurcation, A New Mechanism of Biological Energy Coupling. Chem Rev 118:3862-3886.

18. Peters JW, Beratan DN, Bothner B, Dyer RB, Harwood CS, Heiden ZM, Hille R, Jones AK, King PW, Lu $Y$, Lubner CE, Minteer SD, Mulder DW, Raugei S, Schut GJ, Seefeldt LC, Tokmina-Lukaszewska M, Zadvornyy OA, Zhang P, Adams MW. 2018. A new era for electron bifurcation. Curr Opin Chem Biol 47:32-38.

19. Kaster A-K, Moll J, Parey K, Thauer RK. 2011. Coupling of ferredoxin and heterodisulfide reduction via electron bifurcation in hydrogenotrophic methanogenic archaea. Proc Natl Acad Sci 108:2981-2986.

20. Wagner T, Koch J, Ermler U, Shima S. 2017. Methanogenic heterodisulfide reductase (HdrABC-MvhAGD) uses two noncubane [4Fe-4S] clusters for reduction. Science (80- ) 357:699-703. 
21. Venceslau SS, Stockdreher Y, Dahl C, Pereira IAC. 2014. The "bacterial heterodisulfide" DsrC is a key protein in dissimilatory sulfur metabolism. Biochim Biophys Acta - Bioenerg 1837:1148-1164.

22. Szklarczyk D, Gable AL, Lyon D, Junge A, Wyder S, Huerta-Cepas J, Simonovic M, Doncheva NT, Morris $\mathrm{JH}$, Bork $\mathrm{P}$, Jensen $\mathrm{L}$, Mering $\mathrm{C}$ von. 2019. STRING $\mathrm{v} 11$ : protein-protein association networks with increased coverage, supporting functional discovery in genome-wide experimental datasets. Nucleic Acids Res 47:D607-D613.

23. Sridhar J, Eiteman MA, Wiegel JW. 2000. Elucidation of Enzymes in Fermentation Pathways Used by Clostridium thermosuccinogenes Growing on Inulin. Appl Environ Microbiol 66:246-251.

24. Rubin-Blum M, Dubilier N, Kleiner M. 2019. Genetic Evidence for Two Carbon Fixation Pathways (the Calvin-Benson-Bassham Cycle and the Reverse Tricarboxylic Acid Cycle) in Symbiotic and Free-Living Bacteria. mSphere 4.

25. Tran QH, Bongaerts J, Vlad D, Unden G. 1997. Requirement for the proton-pumping NADH dehydrogenase I of Escherichia coli in respiration of NADH to fumarate and its bioenergetic implications. Eur J Biochem 244:155-60. 
A

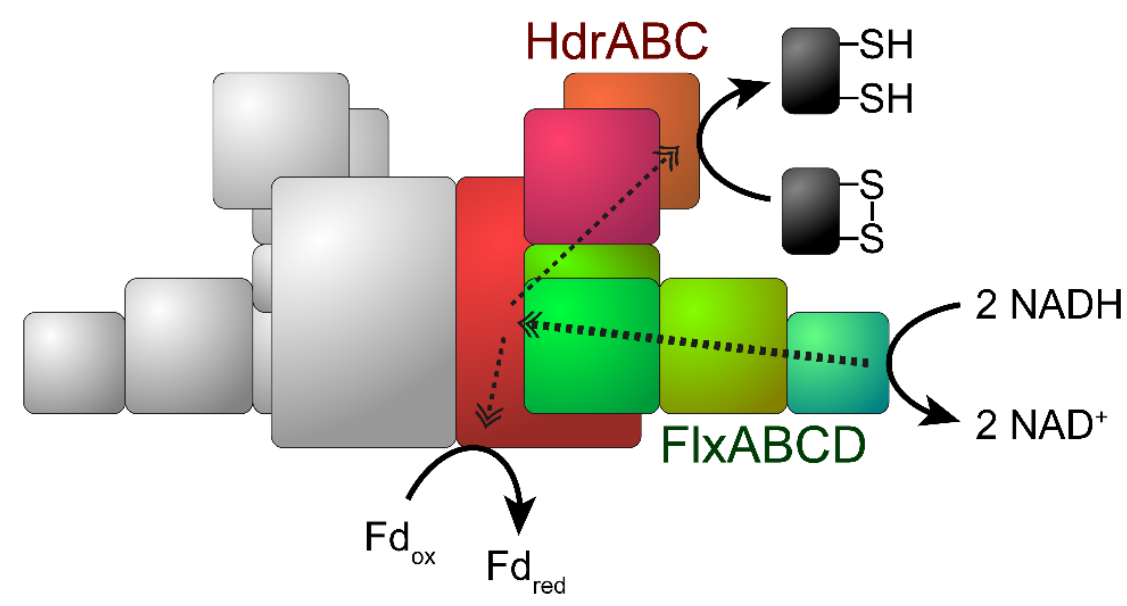

B

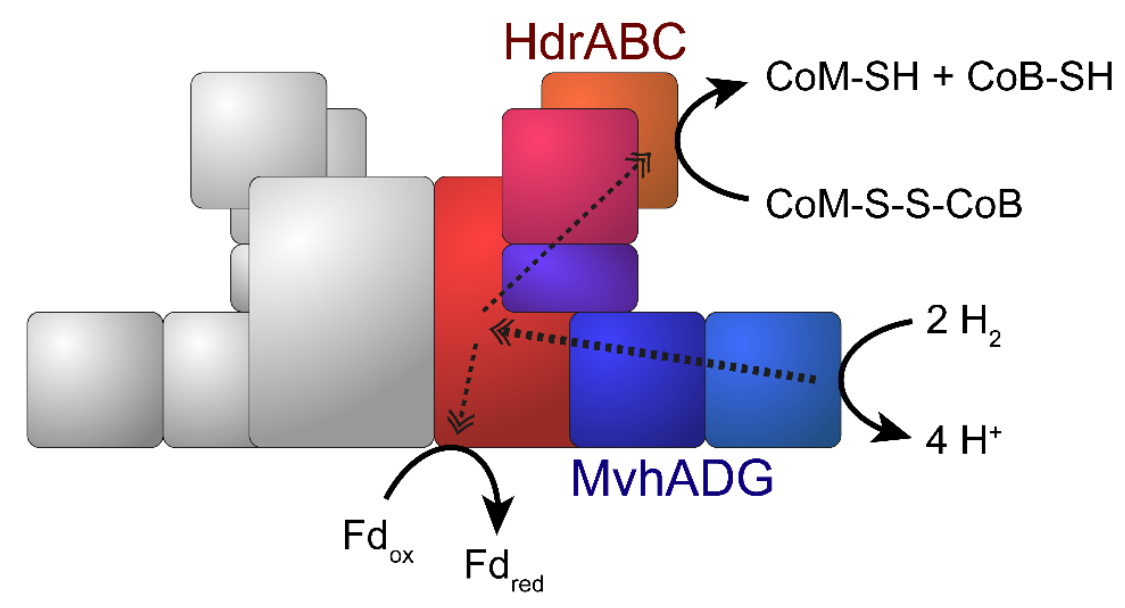

Figure S1. (A) Proposed mechanism of the FlxABCD-HdrABC complex, and (B) the MvhADG-HdrABC complex. Arrangement of the subunits is an interpretation based on the crystal structure of the MvhADG-HdrABC complex, which forms a large dimer of two hetero-hexamers (20). Dashed arrows indicate the flow of electrons. 

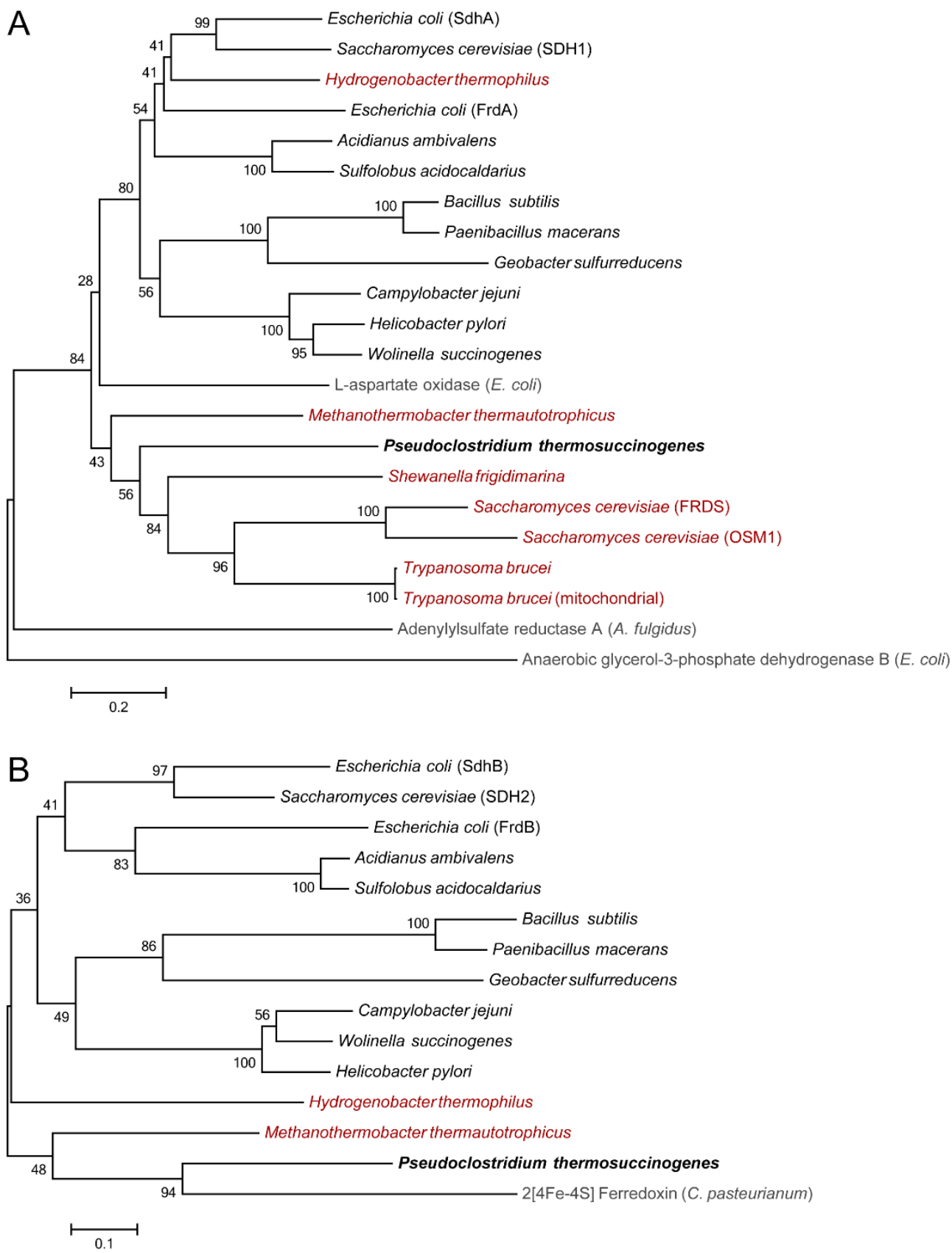

Figure S2. (A) Neighbor joining phylogenetic tree of amino acid sequences from different fumarate reductase flavoprotein subunits. Sequences of the related I-aspartate oxidase, anaerobic glycerol-3-phosphate dehydrogenases, and adenylyl-sulfate reductases are included as well. (B) Neighbor joining phylogenetic tree of amino acid sequences from different fumarate reductase iron-sulfur subunits. Sequence of a ferredoxin, that also contains the $4 \mathrm{Fe}-4 \mathrm{~S}$ ironsulfur cluster is included as well. Reference numbers to the used sequences can be found in the material and methods section. Marked in red are the known soluble fumarate reductases, and in grey are different, but evolutionary related enzymes. The amino acid sequences used for the phylogenetic trees were retrieved from the NCBI database. The following sequences were used for A: CAA06780.1, P08065.4, NP_281599.1, AAC77114.1, AAC73817.1, NP_952230.1, WP_000705948.1，BAG70309.1，CAA04398.1，CAA69872.1，AAB64995.1，AAB59346.1， AAA35026.1， P0C278.1， AAY80343.1, AAN40014.1, AAX20163.1, P17412.3, PNT92608.1, P10902, P13033, O28603. And the following for B: CAA06781.1, NP_390721.1, NP_281600.1, AAC77113.1, AAC73818.1, NP_952231.1, AAC46065.1, WP_012963621.1, CAA04399.1, CAA69873.1, AAS56515.1, P17596.1, PNT92609.1, P00195, Q55389. The sequences are all from enzymatically studied SDHs and FRDs, according to the selection made by Miura et al. (2008) (16). Sequences were aligned using ClustalW with the standard settings in MEGA6. The tree was constructed using the neighbor-joining method in MEGA6, using a bootstrap test with 1,000 replicates. 